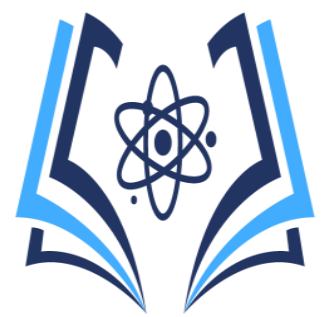

\title{
Humanización del cuidado en las intervenciones de enfermería a pacientes COVID-19 en el servicio de emergencia Hospital Básico San Andrés
}

\section{Humanization of care in nursing interventions for COVID-19 patients in the emergency service Hospital Básico San Andrés Humanización del cuidado en las intervenciones de enfermería}

Delia Georgina Bravo Bonoso. Mg ${ }^{(1)}$, delia.bravo@ unesum.edu.ec

Doris Susana Delgado Bernal. Mg ${ }^{(2)}$, doris.delgado@ unesum.edu.ec

1,2 Universidad Estatal del Sur de Manabí.

Erick Ismael Álava Bravo ${ }^{(3)}$, ismaelalava16@gmail.com

${ }^{3}$ Instituto Superior Universitario Portoviejo

Washington Felipe Álava Castro. Tnglo. ${ }^{(4)}$, junior190179@ hotmail.com

Contacto: delia.bravo@unesum.edu.ec

Recibido: 20-07-2021

Aprobado: 02-08-2021

\begin{abstract}
Resumen
El déficit de humanización en las intervenciones de enfermería, es el principal problema que refleja Hospital Básico San Andrés de Flavio Alfaro en la atención que se brinda al usuario, dejando como consecuencia insatisfacción en la calidad del cuidado que ofrece el personal de enfermería. Se estableció como objetivo general evaluar la humanización del cuidado en las intervenciones de enfermería a pacientes Covid19 en el servicio de emergencia, realizando un estudio analítico- descriptivo no experimental, se aplicó técnicas empíricas como encuestas al personal de enfermería y a usuarios del servicio
\end{abstract}

de emergencia. La muestra seleccionada fue aleatoria simple por muestreo no probabilísticos conformada por 23 enfermeros y 60 usuarios. Los resultados demuestran que el déficit de humanización se da por el exceso de la jornada laboral causando deterioro en la calidad de atención asociándose significativamente al estrés laboral que desarrolla el personal, En base a los resultados obtenidos se concluye que se debe incrementar el personal y reestablecer los horarios de trabajo con el fin de brindar atención de calidad y calidez, estableciendo un mayor compromiso ante los usuarios.

Palabras clave: ser humano, déficit de humanización, empatía e integralidad.

Abstrac

The lack of humanization in nursing interventions is the main problem reflected in the San Andrés de Flavio Alfaro Basic Hospital in the care provided to the user, leaving as a consequence dissatisfaction in https://revistas.itsup.edu.ec/index.php/higia 
the quality of care offered by the nursing staff. The general objective was to evaluate the humanization of care in nursing interventions for Covid-19 patients in the emergency service, conducting a nonexperimental descriptive-analytical study, applying empirical techniques such as surveys to nursing staff and users of the nursing service. emergency. The selected sample was simple random by nonprobability sampling made up of 23 nurses and 60 Keywords: human being, humanization deficit, empathy and integrality

\section{Introducción}

La humanización del cuidado forma parte fundamental de las intervenciones de enfermería, porque al brindar atención de calidad individualizada y digna, se logra un ambiente de confidencialidad y amabilidad sin distinción alguna, pero en los hospitales aún se evidencia el déficit de humanización en las intervenciones de enfermería, convirtiéndose en el principal problema de muchas instituciones de salud.

La humanización de los cuidados son un problema que requiere ser incluido en los servicios asistenciales debido a que los usuarios y familiares quedan insatisfechos con la atención recibida, recalcan que sus derechos no son respetados y que para ser atendidos requiere mucho tiempo de espera (1)

Si se analiza la definición de humanización según el diccionario de la lengua española, humanizar no es tan solo hacer el bien a algo o alguien, es también "ser humano, familiar afable a alguien o algo". "Es ablandarse, desenojarse, hacerse benigno". (2), este concepto posibilita que el personal sea más humano, al considerar aspectos como: cultura, religión, identidad étnica y otros factores que deben ser razonados durante su atención.

Humanizar los cuidados comprende la acción y efecto de brindar atención más humana, familiar y cordial. Con el fin de satisfacer las necesidades de cada usuario que sea atendido en un servicio de users. The results show that the humanization deficit occurs due to the excess of the working day causing deterioration in the quality of care, significantly associated with the work stress developed by the staff. Based on the results obtained, it is concluded that the staff should be increased and reestablished working hours in order to provide quality care and warmth, establishing a greater commitment to users.

salud brindando cuidados dignos y éticos de acuerdo al contexto de cada persona. (3)

Desde la aparición del primer caso de Covid19 en nuestro país, los sistemas de salud, se enfrentaron a un agente desconocido por su etiología, su fisiopatogenia, al pasar el tiempo fueron apareciendo nuevos signos y síntomas que complejizaba la atención y por ende colapsaba las unidades operativas de salud, dejando a la vista la falta del recurso humano, físico, económico, la falta de medicamentos y de equipos de orden primario para la atención de los casos graves, complicando así una atención de calidad y calidez.

"Según Bermejo: humanizar es una cuestión ética, que tiene que ver con los valores y con la búsqueda del bien de la persona a la que se atiende”. (4) todas las personas requieren atención, el personal de salud debe respetar sus derechos mostrar valores y ética adquirida durante su profesión, que desde un direccionamiento estratégico fortalece su responsabilidad con el usuario y familia.

Desde sus inicios el personal de enfermería ha puesto en práctica el cuidado humanizado y este en la actualidad ha ido evolucionando pero que se ha visto afectada por el avance tecnológico, que hace que el personal de enfermería no esté en contacto directo con el usuario especialmente en las instituciones privadas, las unidades de salud pública se ven afectadas por los diversos cambios del sistema interno, infraestructura inadecuada 
Periodo. Julio - Diciembre 2021

para la demanda de usuarios que acuden al área de Vol. 5, Nro. 2, Publicado: 2021-12-31 emergencia, a ello se suma el excesivo trabajo de los profesionales sanitarios puesto que se extiende las 24 horas del día y el trabajo es acelerado induciendo a la búsqueda y práctica del cuidado para preservar y salvar la vida del usuario, dejando en el olvido la humanización e integralidad que deben recibir todos los usuarios. (5)

El vínculo enfermera/paciente es el enlace humanitario que integra el eslabón inagotable del arduo y complicado manejo del paciente en estado agónico. La actitud de la enfermera y su preocupación por brindar satisfacción al paciente; la califica como protectora de una muerte indigna. El servicio de Emergencia, es uno de los servicios que acoge a pacientes más graves o con menos expectativas de curación, en donde la labor como profesionales de enfermería es proporcionar calidad a los cuidados que recibirá el paciente para asegurar las mejores condiciones de vida $\mathrm{y}$ adaptación durante su estancia en el servicio.

Existen una serie de habilidades que los profesionales de enfermería en su diario que hacer le resultan imprescindibles, la presencia de las mismas facilita alinear recursos anímicos que propician mayor habilidad en la resolución de problemas, en fomentar relaciones interpersonales, aumentando así habilidades sociales, lo que ayuda a potenciar el rendimiento laboral y generar defensas para la reacción positiva a la tensión y al stress de cada paciente. Un sistema sanitario humanizado tiene la responsabilidad de estar al servicio de la persona, como función que debe realizar el hombre.

Enfermería tiene como ciencia, la responsabilidad de la calidad del cuidado que proporciona al usuario, institución, ética, leyes, normas de la profesión y contribuye a la práctica en la valoración del cuidado y bienestar del usuario. Cumpliendo un

rol fundamental en el cuidado directo basado en normas y protocolos estandarizados, buscando el bienestar, confort y la recuperación del paciente. (6)

La Comisión on Acreditación of Health Care Organizations, realizó un análisis acerca de la humanización, teniendo en cuenta el ejercicio profesional, para evaluar la ética, la calidad del trabajo y la buena atención clínica, para conocer la importancia de brindar una atención digna $\mathrm{y}$ humanizada en el enfermo, es una acción ética que tiene que ver con los valores que conducen a nuestras acciones en el ámbito de la salud y estos valores nos llevan a plantear políticas para mejorar la calidad de los cuidados y proteger las relaciones enfermero-paciente (7)

La enfermería es una disciplina profesional que abarca cuidados autónomos y en colaboración que se ofrece a las personas, familias y grupos poblacionales, enfermos o sanos, estos cuidados incluyen la promoción de salud, prevención de la enfermedad y los cuidados de las personas en condición de discapacidad e incluso moribundas. Las funciones esenciales del profesional son; la defensa, el fomento de un entorno seguro, la investigación, participación en la política de la salud y en la gestión de los pacientes en los sistemas de salud y la formación. (8)

La Organización Mundial de la Salud define: emergencia sanitaria como la aparición aleatoria de un problema de salud que cause gravedad y que puede generarse en cualquier lugar o actividad que esté realizando, para que exista la necesidad de recibir atención por parte del sujeto que lo sufre o de su familia. La prestación de servicios en el área de emergencia es de acceso libre para todos los usuarios que tenga necesidades de salud, con el fin de resolverlas de manera oportuna a través de procesos lógicos de clasificación como lo es triage. 
Periodo. Julio - Diciembre 2021

Vol. 5, Nro. 2, Publicado: 2021-12-31

A nivel local no se han realizado investigación acerca de la humanización en los cuidados de enfermería puesto que es una problemática que va en aumento y que afecta a los usuarios que llegan a ser atendidos en el Hospital Básico San Andrés de Flavio Alfaro, ya que no reciben atención de calidad donde los derechos de ellos son vulnerados, debido a la demanda de usuarios y el déficit de personal con el que cuenta el servicio de emergencia.

\section{Materiales y Métodos}

A través del presente artículo, se pretende realizar un análisis de contenido de las investigaciones sobre humanización del cuidado en la literatura nacional e internacional. Su proceso de elaboración tuvo varios momentos: la revisión bibliográfica y documental sobre este tema y el análisis del contenido de los mismos, buscando formular inferencias a partir de la identificación de sus características en cuanto a humanización del cuidado en las intervenciones de enfermería y sus determinantes como categoría de análisis.

Se realizó un estudio analítico - descriptivo no experimental con el fin de determinar el déficit de humanización en las intervenciones de enfermería en el servicio de emergencia del Hospital Básico San Andrés de Flavio Alfaro, se aplicó técnicas empíricas como encuestas al personal de enfermería y a usuarios del área de emergencia, considerando la confidencialidad en la información obtenida.

La población fue constituida por 23 enfermeros y 60 usuarios que acudieron al área de emergencia durante el mes de enero de 2021, no se aplicaron técnica de muestreo probabilístico. En atención al área de estudio, la presente investigación se ubica dentro de la categoría documental, la cual depende fundamentalmente de la información que se recoge 
Periodo. Julio - Diciembre 2021

Vol. 5, Nro. 2, Publicado: 2021-12-31

o consulta en documentos; entendiéndose este término, en sentido amplio, como todo aquel material de índole permanente; es decir, al que se puede acudir como fuente o referencia en cualquier momento o lugar, sin que se altere su naturaleza o sentido, para que aporte información o rienda cuentas de una realidad o acontecimiento.

Se seleccionó por criterios de inclusión considerando a todo el personal de enfermería que trabaja en el Hospital Básico San Andrés de Flavio Alfaro y que autorizaron su participación voluntaria en la presente investigación para la aplicación de la encuesta. Los criterios de exclusión fueron personal médico y administrativo, laboratoristas, aquellos que no autorizaron su participación en la investigación y

Tabla 1 Tiempo que laborando en el Hospital que no estuvieron presentes en el momento de la encuesta.

Como instrumento de recolección de datos se utilizó la encuesta dirigida al personal de enfermería y usuarios del área de emergencia, brindando la información necesaria acerca del personal que labora en la institución, Se encuestó a 23 enfermeros que cumplieron criterios de inclusión y se excluyeron a 2 ya que no se presentaron. Los datos recopilados fueron ingresados en el formulario de encuestas luego receptadas en la base de datos de Microsoft Excel 2016, se determinó frecuencia y porcentajes y el análisis de los resultados obtenidos que nos permitió determinar las conclusiones.

\section{Resultados}

\begin{tabular}{lcc}
\hline Alternativa & Frecuencia & Porcentaje \\
\hline 1 a 3 años & 2 & $9 \%$ \\
3 a 5 años & 4 & $19 \%$ \\
5 a 10 años & 6 & $29 \%$ \\
Más de 10 años & 9 & $43 \%$ \\
Total & 21 & 100
\end{tabular}

Fuente: personal de Enfermería del Hospital Básico San Andrés de Flavio Alfaro Elaborado: Autores 
Gráfico 1 Tiempo laborando en el hospital

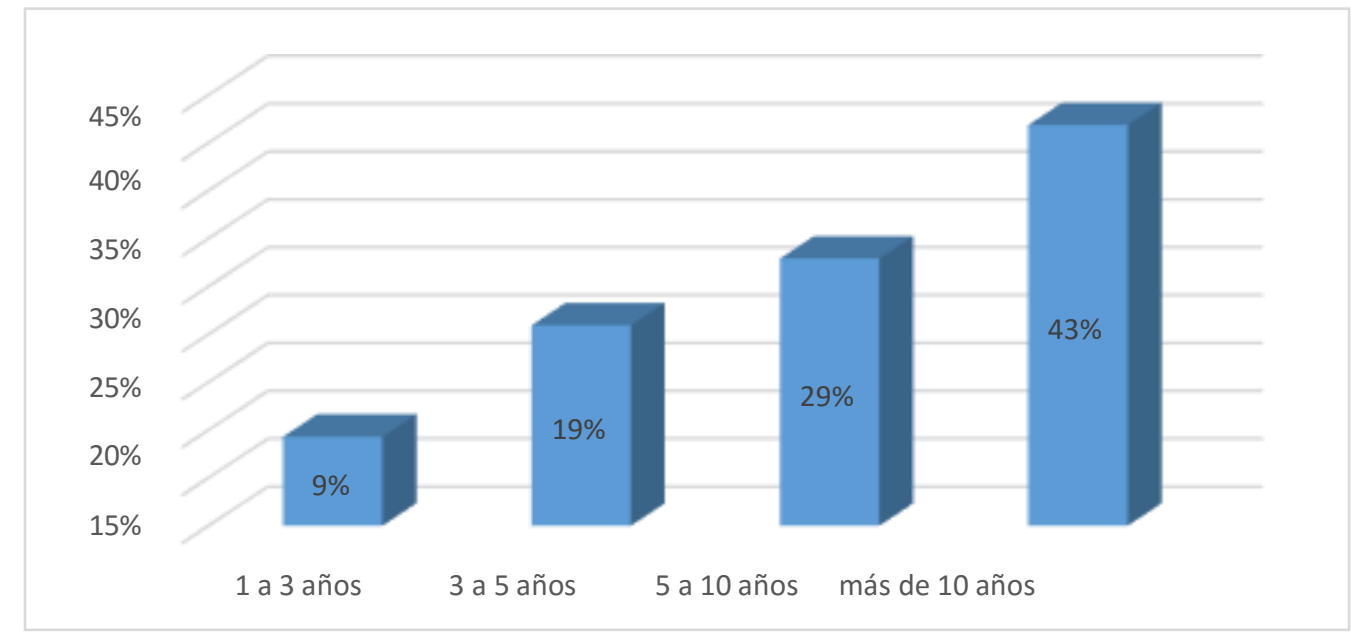

Tabla 2 La jornada laboral dificulta la atención en salud

\begin{tabular}{lcc}
\hline Alternativa & Frecuencia & Porcentaje \\
\cline { 2 - 3 } Siempre & 9 & $43 \%$ \\
A veces & 7 & $33 \%$ \\
Rara vez & 3 & $14 \%$ \\
Nunca & 2 & $10 \%$ \\
Total & 21 & $100 \%$ \\
\hline
\end{tabular}

Fuente: personal de Enfermería del Hospital Básico San Andrés de Flavio Alfaro Elaborado: Autores

Gráfico 2 La jornada laboral dificulta la atención en salud

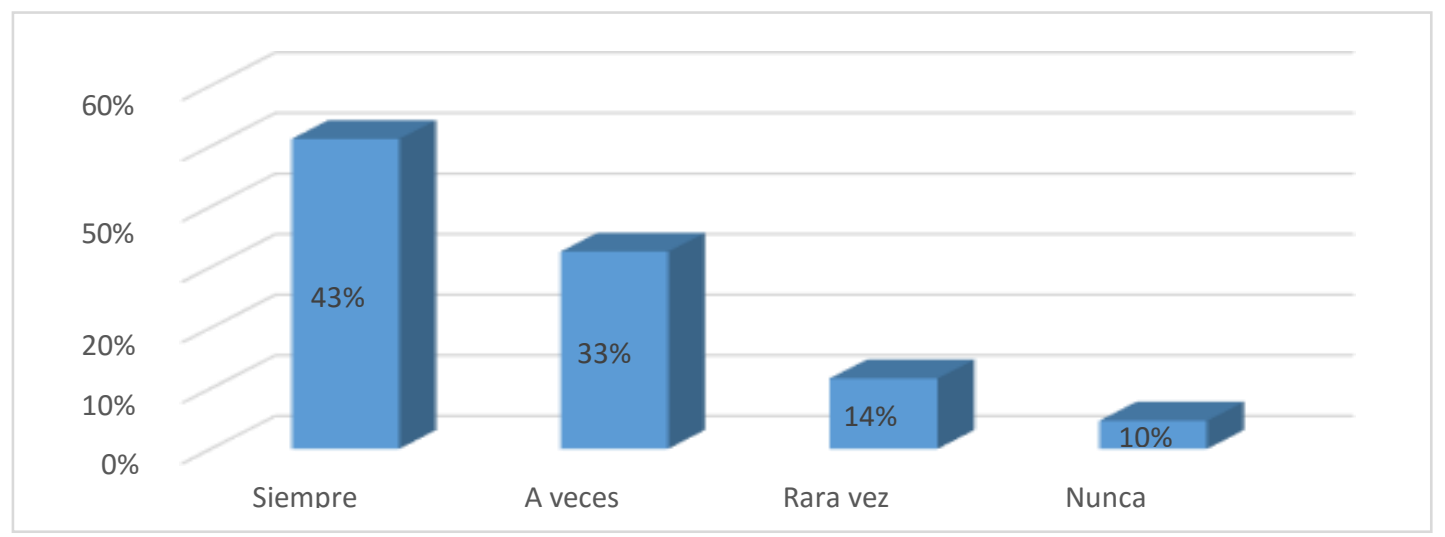

Tabla 3 Recibe maltrato por parte de los usuarios

\begin{tabular}{lll}
\hline Alternativa & Frecuencia & Porcentaje \\
\hline
\end{tabular}


Siempre

A veces

Rara vez

Nunca
Vol. 5, Nro. 2, Publicado: 2021-12-31

$57 \%$

$24 \%$

$14 \%$

$5 \%$

Fuente: personal de Enfermería del Hospital Básico San Andrés de Flavio Alfaro Elaborado: Autores

Gráfico 3 Recibe maltrato por parte de los usuarios

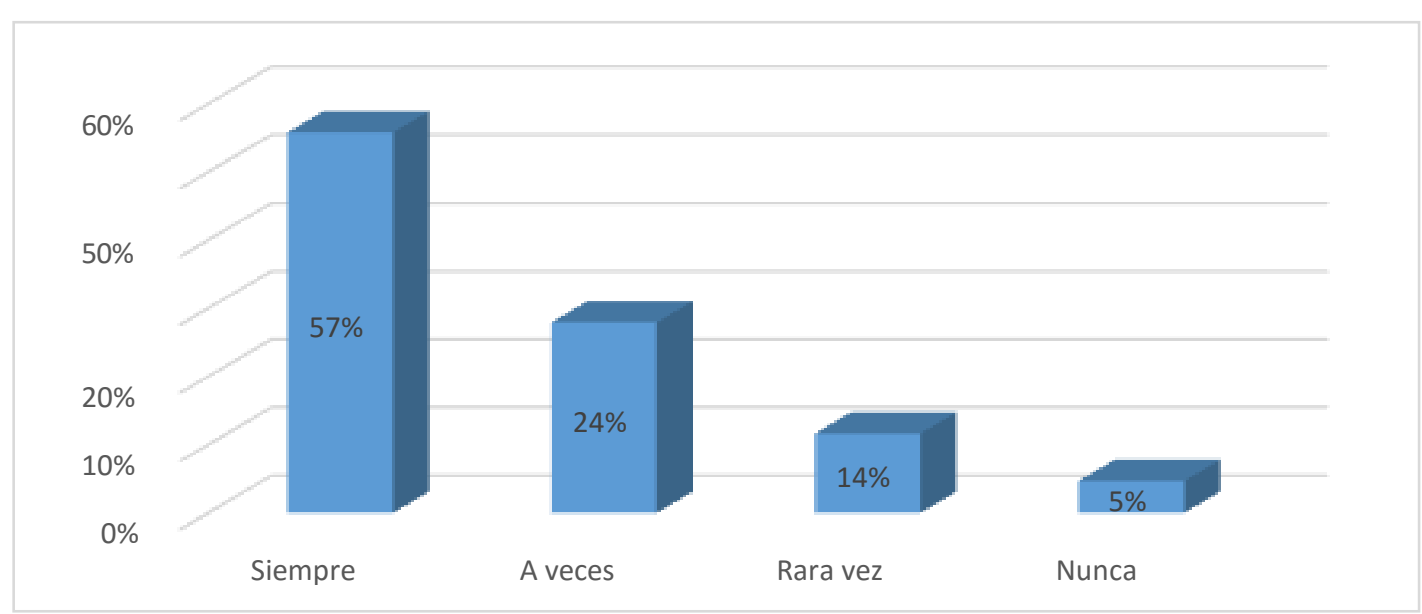

Tabla 4 Capacitaciones acerca del trato humanizado

\begin{tabular}{lcc}
\hline Alternativa & Frecuencia & Porcentaje \\
\hline Siempre & 19 & $90 \%$ \\
A veces & 1 & $5 \%$ \\
Rara vez & 1 & $5 \%$ \\
Nunca & 0 & $0 \%$ \\
Total & 21 & $100 \%$
\end{tabular}

Fuente: personal de Enfermería del Hospital Básico San Andrés de Flavio Alfaro Elaborado: Autores

Gráfico 4 Capacitaciones acerca del trato humanizado

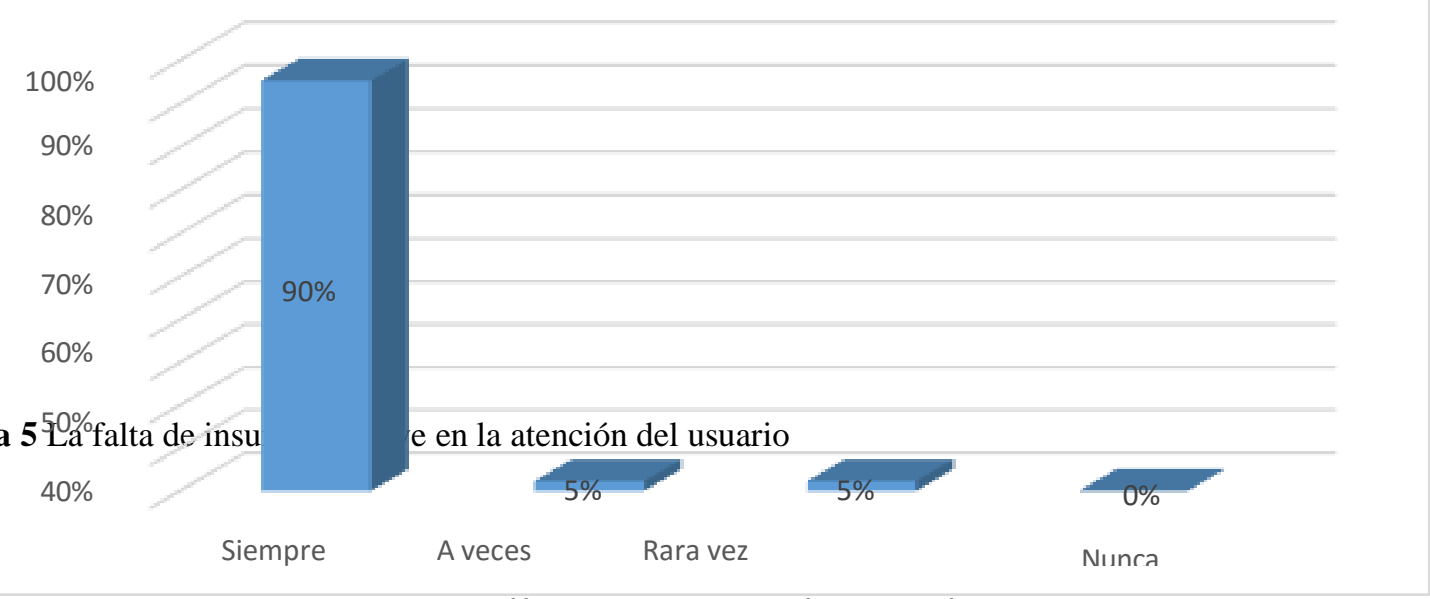

https://revistas.itsup.edu.ec/index.php/higia 


\begin{tabular}{lccc}
\hline Alternativa & Frecuencia & Porcentaje \\
\hline Mucho & & 19 & $90 \%$ \\
Poco & 2 & $10 \%$ \\
Nada & 0 & & $0 \%$ \\
Total & & 21 & $100 \%$
\end{tabular}

Fuente: personal de Enfermería del Hospital Básico San Andrés de Flavio Alfaro Elaborado: Autores

Gráfico 5 La falta de insumos influye en la atención del usuario

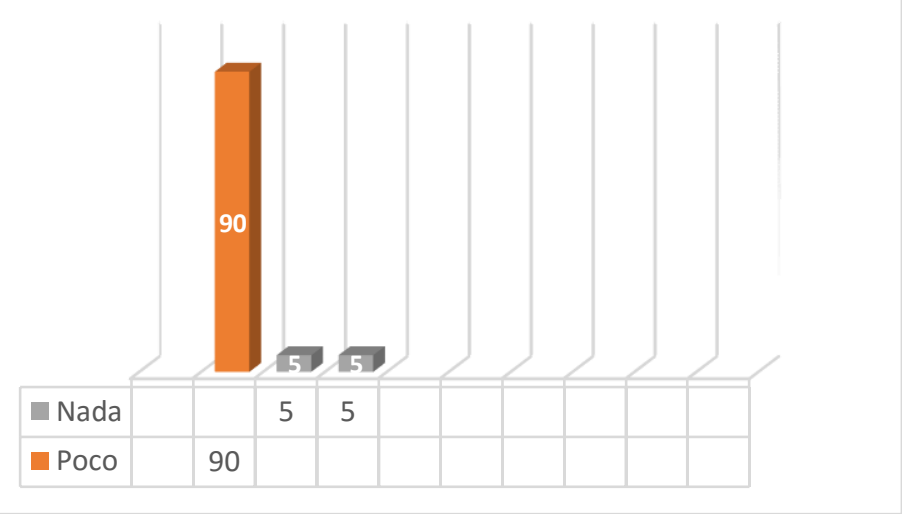

Fuente: personal de Enfermería del Hospital Básico San Andrés de Flavio Alfaro Elaborado: Autores

Tabla 6 La infraestructura dificulta la atención de los usuarios

\begin{tabular}{lll}
\hline Alternativa & Frecuencia & Porcentaje \\
\hline Mucho & 17 & $81 \%$ \\
Poco & 3 & $14 \%$ \\
Nada & 1 & $5 \%$ \\
Total & 21 & $100 \%$
\end{tabular}

Fuente: personal de Enfermería del Hospital Básico San Andrés de Flavio Alfaro Elaborado: Autores

Gráfico 6 La infraestructura dificulta la atención de los usuarios 


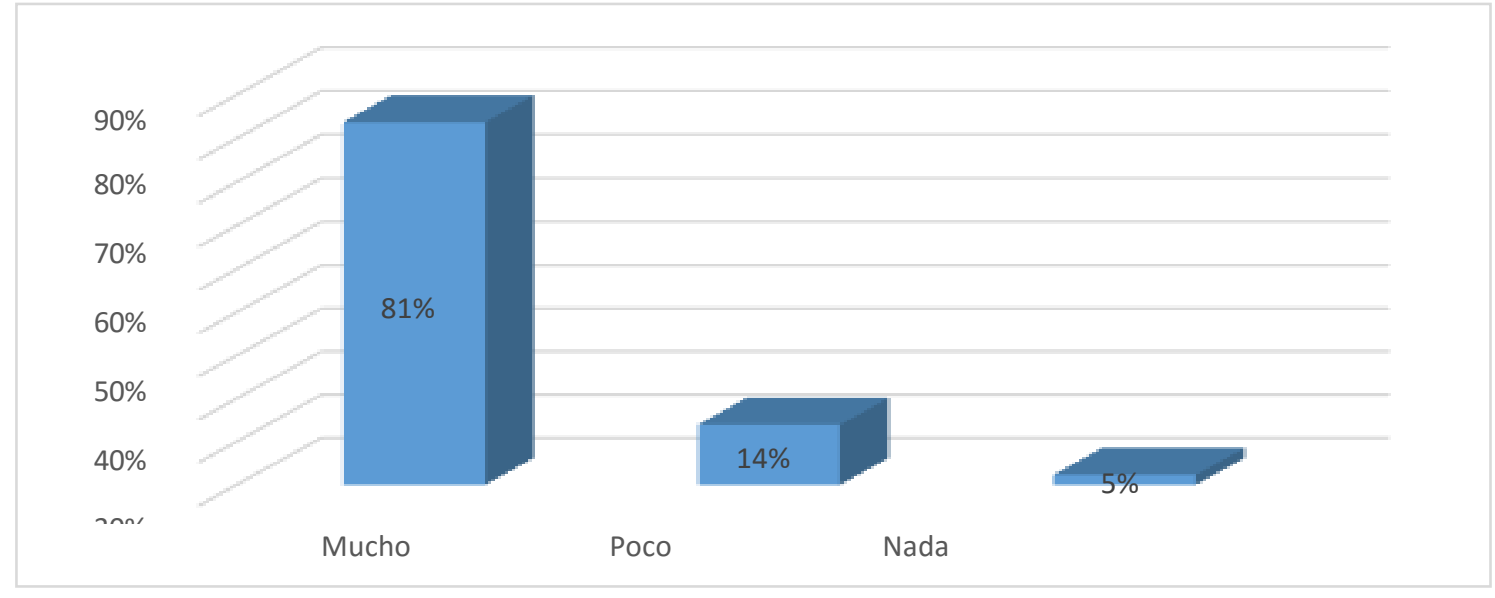

Encuesta a los usuarios

Tabla 7 Cuidados que brinda el personal de enfermería alivian sus necesidades

\begin{tabular}{llll}
\hline Alternativa & Frecuencia & Porcentaje \\
\hline Siempre & 30 & $50 \%$ \\
A veces & 20 & $33 \%$ \\
Rara Vez & 8 & & $14 \%$ \\
Nunca & 2 & & $3 \%$ \\
Total & & 60 & $100 \%$
\end{tabular}

Fuente: usuarios del área de emergencia del Hospital Básico San Andrés de Flavio Alfaro Elaborado: Autores

Gráfico 7 Cuidados que brinda el personal de enfermería alivian sus necesidades

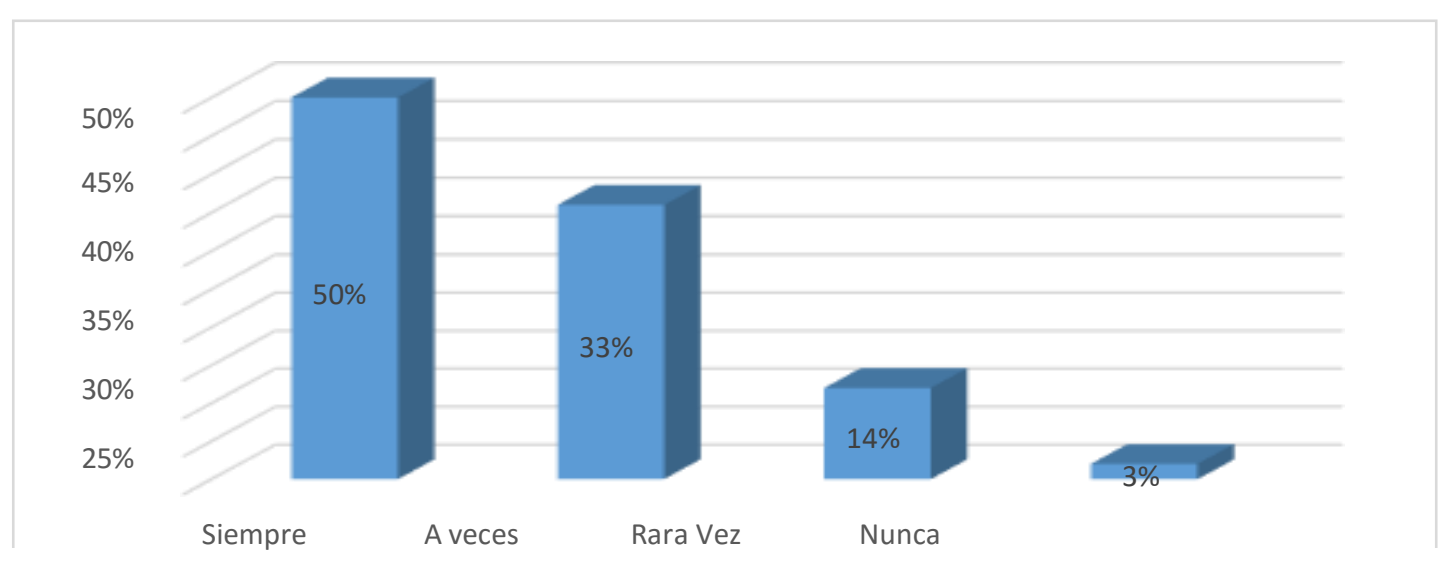

Tabla 8 Calidad de atención que brinda el personal de enfermería

\begin{tabular}{lll}
\hline Alternativa & Frecuencia & Porcentaje \\
\hline
\end{tabular}


Excelente

Muy buena

Buena

Regular

Deficiente

Total
21

16

9

11

3

60
Vol. 5, Nro. 2, Publicado: 2021-12-31 $35 \%$

$27 \%$

$15 \%$

$18 \%$

$5 \%$

$100 \%$

Fuente: usuarios del área de emergencia del Hospital Básico San Andrés de Flavio Alfaro Elaborado: Autores

Gráfico 8 Calidad de atención que brinda el personal de enfermería

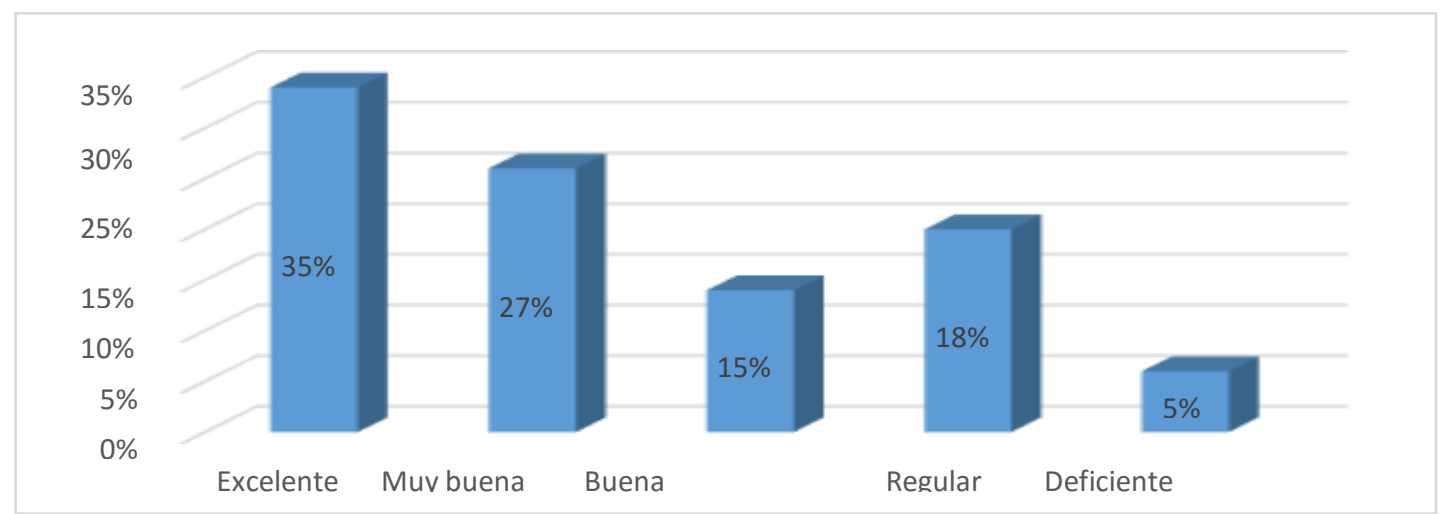

Tabla 9. Generan confianza los cuidados que brinda el personal de enfermería

\begin{tabular}{lll}
\hline Alternativa & Frecuencia & Porcentaje \\
\hline Mucho & 43 & $72 \%$ \\
Poco & 16 & $26 \%$ \\
Nada & 1 & $2 \%$ \\
Total & 60 & $100 \%$
\end{tabular}

Fuente: usuarios del área de emergencia del Hospital Básico San Andrés de Flavio Alfaro Elaborado: Autores 


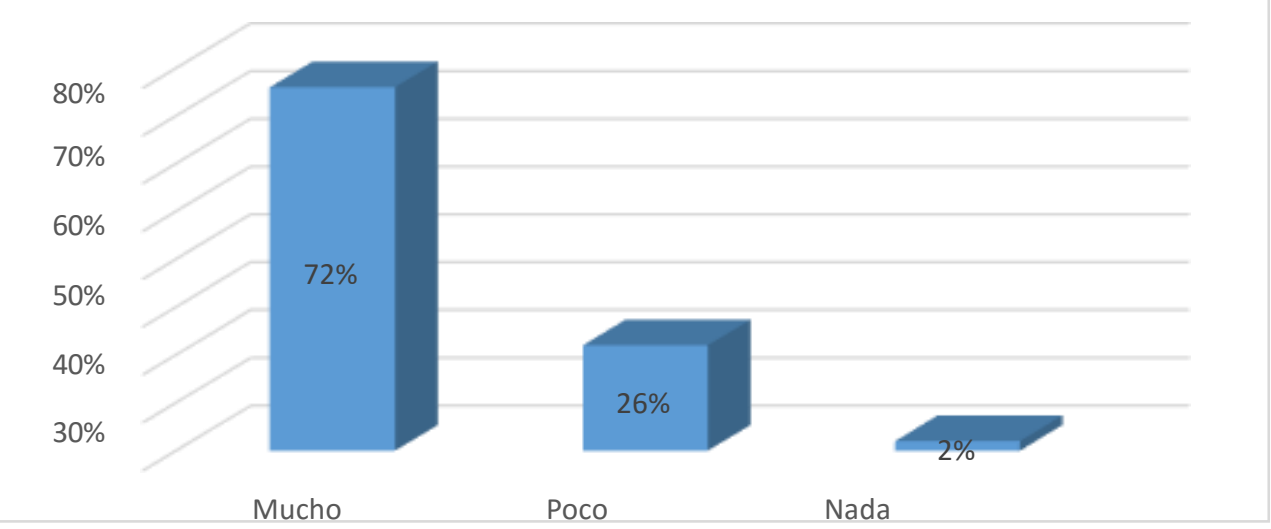

Gráfico 10 Generan confianza los cuidados que brinda el personal de enfermería

\section{Discusión}

La distribución de la jornada laboral dificulta la atención del usuario, el $43 \%$ índico que siempre debido al exceso trabajo que debe realizar durante el día y la limitación de personal lo que conlleva a que no se pueda cumplir con los requerimientos de los usuarios dejando insatisfacción en los cuidados brindados, el 33\% a veces dificulta ya que el personal no cumple con sus turnos ya sea por enfermedad cotidiana o por contagio de la Cocvid-19 que hace que el personal que está laborando debe cubrirlos doblando turno originando deterioro tanto físico como mental en el personal de enfermería poniendo en riesgo la salud del personal o en tales casos por que solo se les asigna un solo día libre después de haber realizado un turno en la noche, el 14\% rara vez, refieren que si afecta las intervenciones que realiza el personal ya que existe cansancio laboral. (Tabla 2) Referente al personal de enfermería recibe maltrato por parte del usuario, el $57 \%$ índico que siempre, esto se debe a los cuidados que se brindan no se los realiza de manera inmediata por la demanda de usuarios que debe atender el personal y se muestran insatisfechos por la atención recibida y porque no logran entender la clasificación de la triada de Manchester de acuerdo a la complejidad de sus signos y síntomas, el $24 \%$ a veces porque el hospital no cuenta con los medicamentos e insumos que tienen que ser adquiridos y no cuentan con el recurso necesario causando inconformidad, el 14\% rara vez (Tabla 3 )

En relación a la aplicación de estrategias que permitan mejor el trato a los usuarios el $30 \%$ indico que se requiere aumentar el personal logrando mejorar la atención que recibe el usuario ya que se bridaría cuidados individualizados y habría interacción enfermera usuario para poder así fomentar un ambiente de calidad y calidez, el 28\% disminución de la jornada laboral, si se realizan turnos de 120 horas el personal no tendría mucha carga laboral lo que favorecía al usuario en el atención brindada, el $23 \%$ realizar turnos de 12 horas, lo que permitirá tener mayores días de descanso favoreciendo al personal y mantener el vínculo familiar y disminuir el estrés laboral que se presenta diariamente, 14\% jornadas integradoras ayude al personal de salud en mejorar su estado físico y mental. (Tabla 6) El personal de enfermería se vería favorecido mejorando los procedimientos de trabajo, salario, los insumos, derechos y capacitación sobre cada uno de las intervenciones a realizar en su área de desempeño con el fin de buscar satisfacción laboral beneficiando al personal de enfermería (13)

En relación a la calidad de atención, se lleva a cabo una serie de acciones inmediatas para que los sistemas de salud presten atención efectiva, segura, 
Periodo. Julio - Diciembre 2021

Vol. 5, Nro. 2, Publicado: 2021-12-31

eficiente, accesible, apropiada y satisfactoria para los usuarios y en pro de la seguridad del paciente y que se cumplan cada una de las necesidades por la que los usuarios llegan a ser atendidos.

La distribución de la jornada laboral dificulta la atención del usuario, el $43 \%$ indicó que siempre debido al exceso de trabajo que debe realizar durante el día lo que conlleva a que no se pueda cumplir con los requerimientos de los usuarios dejando insatisfacción en los cuidados brindados. Para Flyn en un estudio demostró que las altas cargas de trabajo, falta de apoyo en el ambiente de trabajo, se traduce en un deterioro de la calidad de atención y de la satisfacción del usuario lo que se asocia a un aumento significativo de estrés laboral en el personal de enfermería. (14) (Tabla 2)

En la aplicación de estrategias que permitan mejorar el trato humanizado a los usuarios el $30 \%$ indico que se requiere aumentar el personal. Para Shirley Maldonado, el personal insuficiente, déficit de la infraestructura, distribución de los espacios, trabajo mal remunerado, falta de mobiliario causan déficit de atención en los usuarios, afirma que cada institución que brinda cuidados a usuarios debe contar con recursos humanos, materiales $\mathrm{y}$ económicos que ayuden a solventar las necesidades de los usuarios que llegan a ser atendidos y resolver sus problemas de salud. (15)(Tabla 6)

En el análisis acerca de los cuidados que brinda el personal de enfermería al usuario satisfacen las necesidades el $50 \%$ respondió siempre, pero que el trato que reciben no es el indicado ya que no respetan sus derechos. Para Jean Watson el cuidado de enfermería permite lograr un nivel de reconocimiento del usuario y del familiar (16) . La satisfacción de las necesidades básicas en el cuidado humanizado se basa en la individualidad del paciente para poder implementar habilidades $\mathrm{y}$ técnicas propias de enfermería que ayuden a brindar un cuidado oportuno, consciente, creativo frente a las necesidades del otro. (Tabla 8)

\section{Conclusiones}

- El cuidado humanizado históricamente aparece en tiempos de crisis donde el ser humano debe ser tratado como un ser único solventando cada una de sus necesidades, haciendo referencia al proceso de comunicación y apoyo mutuo que debe tener entre la enfermera y el usuario.

- Se evidenció que los usuarios tienen poco conocimiento acerca de trato humanizado, lo que afecta la relación enfermera paciente en la atención directa favoreciendo la deshumanización en el cuidado que brinda el personal de enfermería en el Hospital Básico San Andrés de Flavio Alfaro

- Las barreras que impiden al personal de enfermería brinden cuidado de calidad; es el déficit de personal, la falta de recursos materiales y la infraestructura que no es la adecuada, para solventar cada una de las necesidades por las que acuden los usuarios de la zona norte de Manabí.

- Una de las principales estrategias que el personal de enfermería requiere es el incremento de personal y que se reestablezcan los horarios de trabajo, esto permitirá mejorar la atención contando recursos y talento humano suficiente que ofrezca atención de calidad y calidez. 
Bibliografía

1. Andino C. Bioetica y Humanización de los Servicios de Salud. Revista Colombiana de Bioetica. 2016;: p. 29.

2. Consejeria de Sanidad. Plan de Humanizacion de asistencia sanitaria Madrid: Biblioteca Virtual; 2016.

3. Malpico Tinoco M. PERCEPCIÓN DEL CUIDADO HUMANIZADO DE ENFERMERÍA POR PARTE DEL PACIENTE ADULTO CON ENFERMEDAD ONCOLÓGICA HOSPITALIZADO EN UNAIPS DE IV NIVEL. Mexico:; 2017.

4. Gutierrez Fernandez. La humanización de (en) la Atención Primaria. cielo. 2017;: p. 11.

5. Valenzuela Anguita M. Es posible humanizar los cuidados de enfermeria en los servicios de emergencias. Madrid:; 2015.

6. Rodriguez-Jimenez ,S, Cardenas-Jimenez ,M, Pacheco-Arce AL, Ramirez-Perez ,M. Una mirada fenomenológica del cuidado de enfermería. Scielo. 2014.

7. Santacruz-Bravo JD. Humanización de la calidad en la atención clínica en salud desde la perspectiva centrada en el paciente a partir de la Resolución. Colombia:; 2015.

8. De Arco-Canoles DC, Suarez-Calle K. Rol de los profesionales de enfermería en el sistema de salud colombiano. Universidad y Salud. 2018.

9. Lagoueyte Gómez M. El cuidado de enfermería a los Grupos Humanos. Revista de la Universidad Industrial de Santander. Salud. 2015.

10. Molano Gutiérrez R, Escobar Grisales , García Vargas A, Salazar Molina A, Mejía Venegas , Jiménez Barbosa. Motivos de uso de los servicios de urgencias. ACTA. Odontologico Colombiano. 2016.

11. Duarte V. Humanización de la asistencia médica en el servicio de urgencias: la importancia de los valores en salud. Dialnet. 2014;: p. 16.

12. Savinovich Morán C. Humanización en la atención de enfermería a los usuarios de la Sala de Emergencia del Hospital "Dr. Teodoro Maldonado Carbo", de diciembre 2013 a mayo 2014. Guayaquil:; 2014.

13. Quintana Zavala O. Calidad de vida en el tabajo, personal de enfermeria. Chile:; 2014.

14. Casé R, Susana Lopetegu N. Matrices Progresivas de Raven: efecto Flynn y actualización de baremos. Revista Psicologica de Chile. 2014;: p. 12.

15. Maldonado Hurtado SM. Buenas practicas empresariales para la inclusion laboral de profesionales con discapacidad. Quito:; 2018.

16. Guerrero Ramírez R, Meneses La Riva, De La Cruz-Ruiz. Cuidado humanizado de enfermería según la teoría de Jean Watson,servicio de medicina del Hospital Daniel Alcides Carrión. Lima. Rev enferm Herediana. 2015;: p. 10. 